\title{
Article
}

\section{Using Drones for Art and Exergaming}

Scheible, J, Funk, M, Pucihar, K.C, Kljun, M, Lochrie, Mark, Egglestone, Paul and Škrlj, P

Available at http://clok.uclan.ac.uk/19002/

Scheible, J, Funk, M, Pucihar, K.C, Kljun, M, Lochrie, Mark ORCID: 0000-00027909-8455, Egglestone, Paul and Škrlj, P (2017) Using Drones for Art and Exergaming. IEEE Pervasive Computing, 16 (1). pp. 48-56. ISSN 1536-1268

It is advisable to refer to the publisher's version if you intend to cite from the work. http://dx.doi.org/10.1109/MPRV.2017.4

For more information about UCLan's research in this area go to http://www.uclan.ac.uk/researchgroups/ and search for < name of research Group>.

For information about Research generally at UCLan please go to http://www.uclan.ac.uk/research/

All outputs in CLoK are protected by Intellectual Property Rights law, including Copyright law. Copyright, IPR and Moral Rights for the works on this site are retained by the individual authors and/or other copyright owners. Terms and conditions for use of this material are defined in the policies page.

\section{CLoK}

Central Lancashire online Knowledge www.clok.uclan.ac.uk

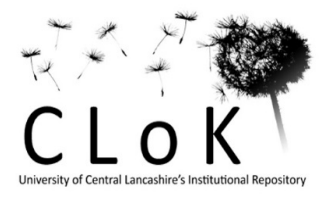




\section{A Moving Projector Platform: Interactive Context Aware Projections with Drones for Exergaming}

Klen Čopič Pucihar, University of Primorska, FAMNIT Glagoljaška 8, 6000 Koper, Slovenia, klen.copic@famnit.upr.si

Matjaž Kljun, University of Primorska, FAMNIT Glagoljaška 8, 6000 Koper, Slovenia, matjaz.kljun@upr.si

Mark Lochrie, Media Innovation Studio, University of Central Lancashire, PR1 2HE Preston, UK, mlochrie@uclan.ac.uk

Paul Egglestone, Media Innovation Studio, University of Central Lancashire, PR1 2HE Preston, UK pegglestone@uclan.ac.uk

Peter Škrlj, University of Primorska, FAMNIT Glagoljaška 8, 6000 Koper, Slovenia, peter.skrlj@student.upr.si 


\section{Introduction}

Digitally augmented public spaces and surfaces are ever more present in our daily lives. Examples (see Figure 1) range from art installations (e.g. Deep Time projection mapping at the Edinburgh International Festival in 2016), to installations for raising public engagement (e.g. projection mapping on National Monument of Scotland on Calton Hill in 2015 made by mclcreate), to displays for promotion (e.g. public information display panels at the Samsung Delight promotion hall in Seoul), and displays for supporting community awareness (e.g. WrayDisplay digital community noticeboard [1]). These systems are best used where static or a place specific setup is required. For this reason they are usually confined to a single location and/or event, which is not always desired.
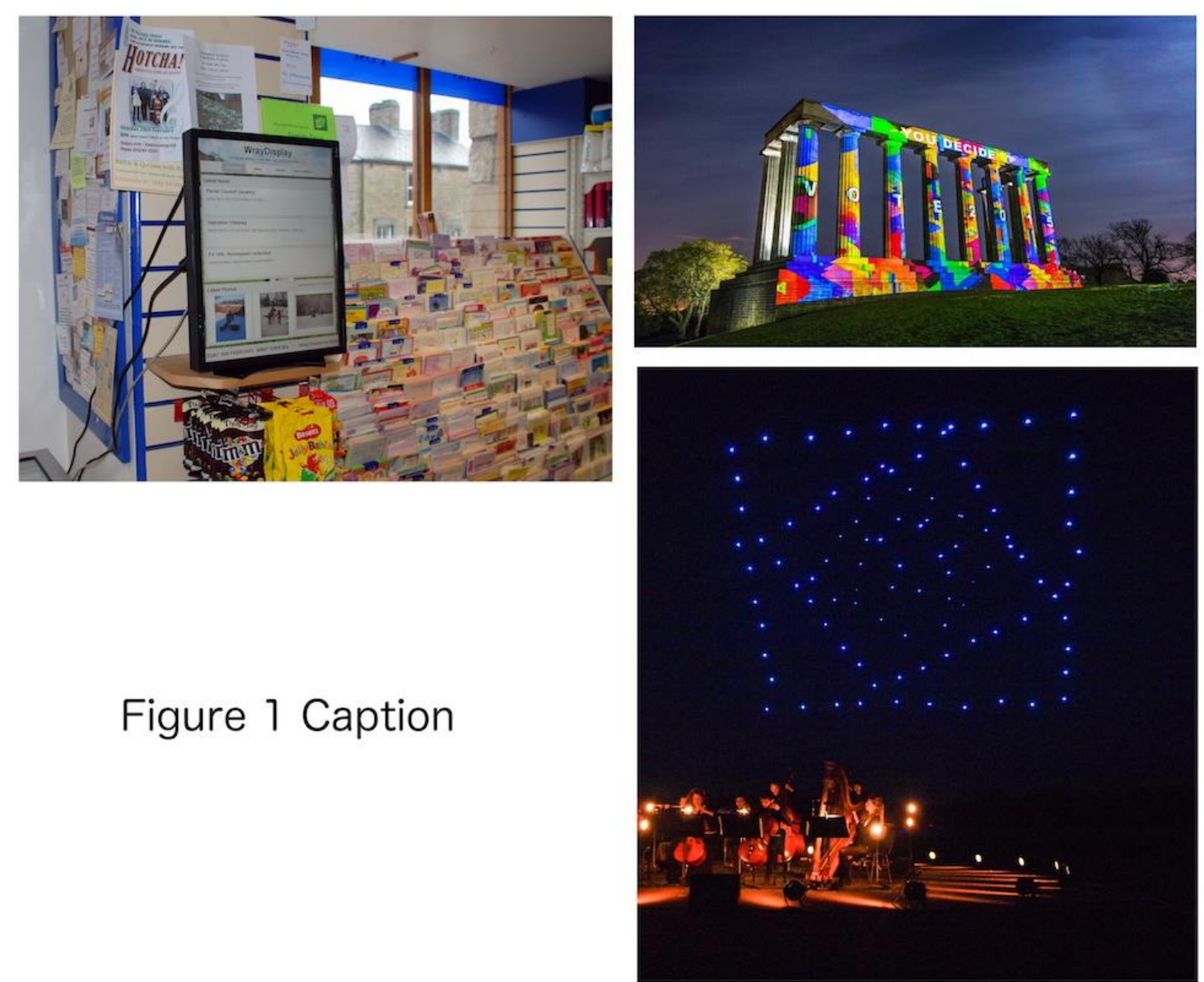

Figure 1: Left top: WrayDisplay digital community noticeboard (courtesy of Nick Taylor; used with permission). Right top: mclcreate public engagement installations displaying a message on the National Monument of Scotland ${ }^{1}$ (photo by Craig Robertson, courtesy of the mclcreate; used with permission). Right bottom: Intel's and Ars Electronica's aerial entertainment with choreographed lightened up drones displaying a desired content based on drones' formation (photo by Martin Hieslmair, courtesy of Ars Electronica, used with permission).

Recently, research has focused on various technology setups of moving displays as a communication and interaction medium in public and semi-public domains for various contexts such as displaying emergency information, supporting social interactions or displaying information at difficult to reach places. In particular, Unmanned Aerial Vehicles (UAVs), or drones have further fueled research and

\footnotetext{
${ }^{1}$ Art collective involved in the projection created by Projector Club.
} 
technology possibilities of moving displays in order to alleviate them from static environments and facilitate content projection anywhere at anytime.

Early examples of such drone based moving displays are the so called Midair displays (where information is displayed on a screen attached to a drone), Displaydrone (where information is projected on arbitrary surfaces by a drone carried projector), and Flying display [2] and In-Situ-DisplayDrone (where a drone carries a projector to display information on a canvas carried by the same or another drone $)^{2}$. Another example are multiple drone formations such as Intel's and Ars Electronica's aerial entertainment with choreographed lightened up drones displaying a desired content based on drones' formation (see Figure 1), and BitDrones [3] that create a "self-levitating programmable matter" (enabling users to physically interact with multiple drones to create basic designs in 3D space).

Despite a vibrant research activity on moving pervasive displays, existing systems implement limited or no interactivity. Most commonly, co-located users are able to socially interact by sending content to the system (such as photos on WrayDisplay or short text messages on DisplayDrone), which is then displayed or projected for other users to see. Moreover, the displays described have no or little awareness of colocated users and their movements, hence, are not able to adapt displayed content to different situations.

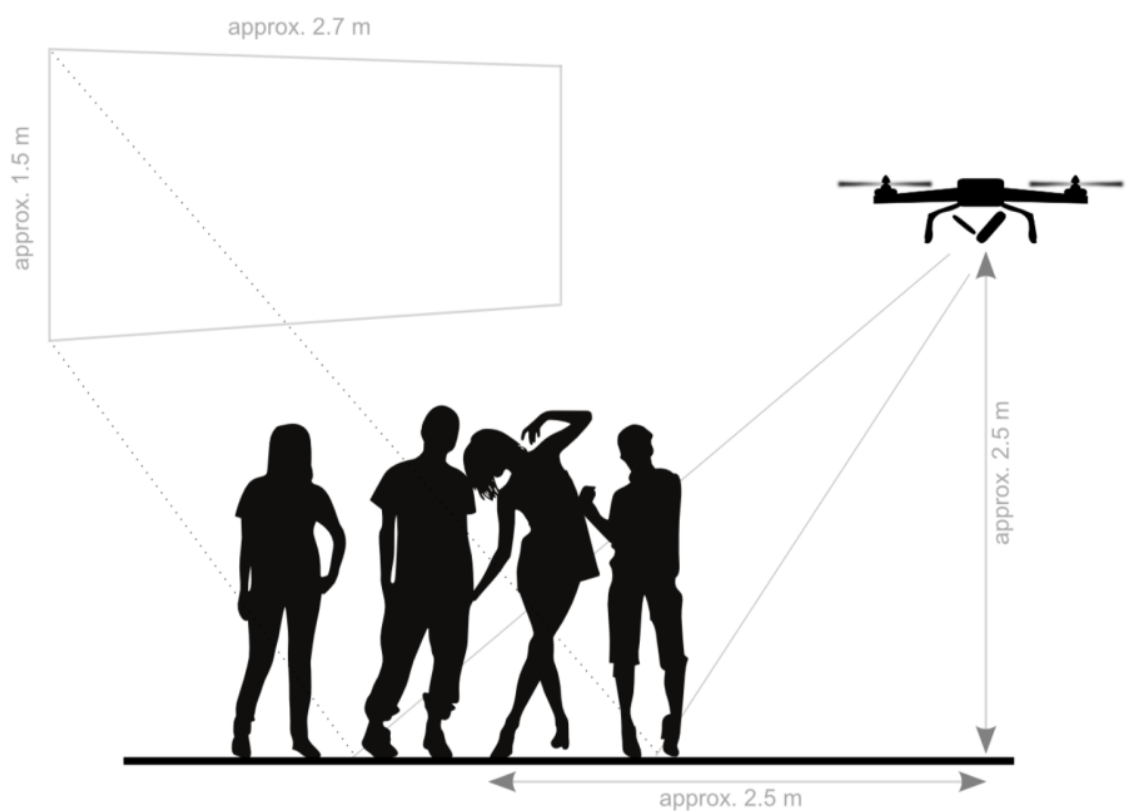

Figure 2. Moving projector platform is flying at a safe distance from players (approx. $2.5 \mathrm{~m}$ from ground up and $2.5 \mathrm{~m}$ from center of projection in horizontal direction distance). Such setup creates a playing area of about $1.5 \mathrm{~m} \times 2.7 \mathrm{~m}$.

To address the lack of interactiveness we are working on a Moving Projection Platform (see Figure 2) that is able to project the content on arbitrary surfaces while tracking user interaction within the displayed content as well as around it. Moving Projection Platform thus brings pervasive displays towards the ultimate state where interaction with content displayed on arbitrary objects becomes object specific [4]. Exergames are one scenario where the mobility and rich interaction possibilities are fundamental and create an opportunity to advance research of human-drone interaction in the context of street games.

Street games (i.e. hoping rope) and sidewalk chalk games (e.g. hopscotch) have been pervasive on the streets for centuries. Lately, games have been returning to streets in the form of Location Based Games (LBG) commonly implemented using mobile phone technology. LBG's have recently showcased extreme

\footnotetext{
${ }^{2}$ More about Midair displays, Displaydrone and In-Situ-DisplayDrone can be read in the article of this issue titled "Using Drones in Human-Computer Interaction to create Flying Displays and Flying Viewports for art making"
} 
commercial success (e.g. Pokemon Go); however, the dependence of games on what is shown on the screen, be it the object of play or the instructions of the game, often contributes to the players inability to fully immerse into the game by disconnecting the user from the environment and interrupting communication between players within the co-located multiplayer game [5]. With the Moving Projector Platform the environment becomes an interactive pervasive display, which allows players to focus on their surroundings and be more attentive of other players in multiplayer games situations. Additionally, the ability to move the gaming platform during the game also enables us to explore new forms of interactive entertainment and dynamic visual experiences in co-located settings.

\section{The System}

The Moving projection platform (see Figure 2) called StreetGamez is designed as an open platform that can facilitate various types of street and chalk games utilizing a drone as a method for delivering the mounted game infrastructure to a requested location. Besides flying to the location of the game, the platform performs three vital tasks, namely: (i) project graphics onto arbitrary surfaces, (ii) enable interaction within the projected surface through motion tracking of players, and (iii) enable interaction through players' mobile device (e.g. calling the platform to a desired location, selecting games, etc.).

The system is based on the Google Tango ${ }^{3}$ device which is used for tracking player movements as well as rendering the game graphics. Presently the system is limited to projections on horizontal planar surfaces where the system currently only tracks players' feet (see Figure 3). While the Tango point cloud detection is limited on average to 10 FPS, more responsive detection is done by tracking feedback zones inside the captured image using Tango's rgb camera. The system distinguishes between players through colour tracking, hence in the current version unique footwear colour is required in order to play a multiplayer game. The tracking system utilizes depth camera and colour based tracking techniques. In order to enable easy integration with new and existing games, a decision was made to make our system compatible with Unity game engine and offer our player tracking and game projection solution as a Unity template better know as Unity prefab 4 . We decided on this in order to enable easy integration when modifying existing or creating new games that are compatible with our platform.

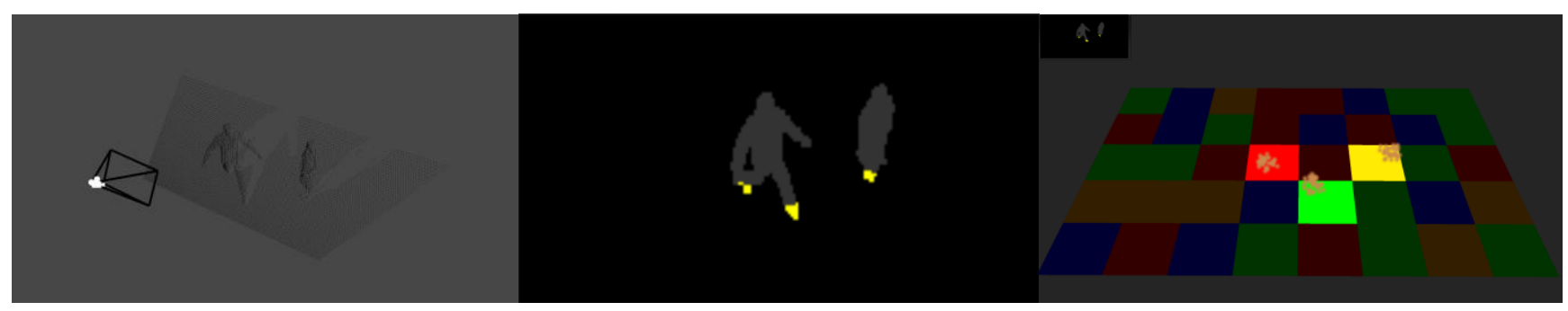

Figure 3: Left: Depth map rendered. Center: Feedback detection with grouping points of interest near the floor surface. Right: Player feet positions projected onto interactive surface.

There are still some technical limitations to the proposed system common to most drone based systems such as limited flight time, potential harming of colocated players, projector's brightness and compliance with in-country and international aviation regulations.

\footnotetext{
3 http://get.google.com/tango/developers/

4 The Unity prefab acts as a template from create GameObject instances in the scene.
} 


\section{Games}

Introducing and showcasing new modes of interaction and system abilities is important for inspiring other researcher, game and creative industry to further develop technology and define its popular forms. In light of this goal our system introduces three game scenarios, namely: Static-hover, On-the-move and Drona-as-game-element [6].

Static-hover is an example of a classical projected game scenario where graphical game elements are projected onto a physical environment and become interactive through the motion tracking of players. In this setup the drone hovers at the fixed position and facilitates the game being played on the ground (e.g. whack-a-mole, hopscotch, etc.). However, in contrast to fixed projected games (e.g. Augmented Climbing [7] where a projected chainsaw is introduced to a climbing wall and grips can become interactive) re-positioning the playing surface presents no difficulty.

On-the-move is a novel game paradigm where the drone moves with players and facilitates the game whilst on the move. One example of such game would be LBG (i.e. treasure hunt) where the game is aware of the context where it is being played, hence in this case projections become context aware. The drone could change its position in response to the players' movements providing human-drone interaction.

Drone-as-game-element is the next novel game paradigm. Here the drone becomes the game element where it takes an active role as a player (e.g. hide and seek, tag). This can yet again facilitate new interaction possibilities for human-drone interaction.

\section{Further remarks}

Entertainment and gaming industry already explores innovative approaches in the area of game design enhanced by emerging technologies such as drones. Examples are Lumi quadcopter by WowWee ${ }^{5}$ (offering various games such as controlling drone illumination colour using a mobile phone), Parrot drones in combination with AR.PURSUIT augmented reality game (in which one player pursues a drone of another player in order to bring it down with virtual machine guns and missiles visible through the mobile device screen), and Air Hogs Connect game (where players control a real-world drone as they progress through an AR videogame visible on the tablet screen). Novel games with drones such as aerial combat and drone racing have also seen many competitors and spectators at various league events.

Drones in the context of gaming present a technology already accepted by early adopters and provide an emerging and exciting research area for studying human-drone interaction in entertainment. However, in contrast to our Moving Projection Platform where drones are autonomous and provide digitally augmented physical environment, current games require users to remotely control the drone's movements in space by mobile devices or remote controls.

Our three proposed game scenarios free players from the need to carry additional gaming equipment allowing them to focus on the game surroundings and other players in the game, which is very similar to real life situations where people do not carry special equipment to be able to interact with pervasive displays and follow the surroundings. Studying advanced interactive and movable game environments immersed into players' surrounding made possible by our platform can thus provide knowledge that can be applicable in other moving pervasive display settings (e.g. adaptive escape route planning in an

${ }^{5} \mathrm{http}: / /$ wowwee.com/lumi 
emergency situation that can be based on people's (re)actions as well as the changes in the environment).

\section{REFERENCES}

[1] Taylor, Nick, and Keith Cheverst. "Supporting community awareness with interactive displays." Computer 45.5 (2012): 26-32.

[2] Nozaki, Hiroki. "Flying display: a movable display pairing projector and screen in the air." CHI'14 Extended Abstracts on Human Factors in Computing Systems. ACM, 2014.

[3] Gomes, Antonio, et al. "BitDrones: Towards Using 3D Nanocopter Displays as Interactive SelfLevitating Programmable Matter." Proceedings of the $2016 \mathrm{CHI}$ Conference on Human Factors in Computing Systems. ACM, 2016.

[4] Huber, Jochen, et al. "LightBeam: interacting with augmented real-world objects in pico projections." Proceedings of the 11th International Conference on Mobile and Ubiquitous Multimedia. ACM, 2012.

[5] Gradinar, Adrian, et al. "Designing for the dichotomy of immersion in location based games." Proceedings of the Foundations of Digital Games. SASDG, 2015.

[6] Kljun, Matjaž, et al. "StreetGamez: A Moving Projector Platform for Projected Street Games." Proceedings of the 2015 Annual Symposium on Computer-Human Interaction in Play. ACM, 2015.

[7] Kajastila, Raine, Leo Holsti, and Perttu Hämäläinen. "The Augmented Climbing Wall: High-Exertion Proximity Interaction on a Wall-Sized Interactive Surface." Proceedings of the $2016 \mathrm{CHI}$ Conference on Human Factors in Computing Systems. ACM, 2016. 